\title{
STOCHASTIC FINITE ELEMENT ANALYSIS USING POLYNOMIAL CHAOS
}

\author{
S. DRAKOS, G.N. PANDE \\ International Center for Computational Engineering, Rhodes, Greece
}

\begin{abstract}
This paper presents a procedure of conducting Stochastic Finite Element Analysis using Polynomial Chaos. It eliminates the need for a large number of Monte Carlo simulations thus reducing computational time and making stochastic analysis of practical problems feasible. This is achieved by polynomial chaos expansion of the displacement field. An example of a plane-strain strip load on a semi-infinite elastic foundation is presented and results of settlement are compared to those obtained from Random Finite Element Analysis. A close matching of the two is observed.
\end{abstract}

Key words: foundation settlements, stochastic finite element, polynomial chaos

\section{INTRODUCTION}

The design of foundations of structures requires an assessment of settlement which is generally difficult because of the uncertainty and spatial variability of the properties of soil materials. Various forms of uncertainties arise which depend on the nature of geological formation, the extent of site investigation, the type and the accuracy of design calculations, etc. In recent years, there has been considerable interest amongst engineers and researchers in the issues related to quantification of uncertainty as it affects safety, design as well as the cost of projects.

A number of approaches using statistical concepts have been proposed in geotechnical engineering in the past 25 years or so. These include the Stochastic Finite Element Method (SFEM) (Phoon et al. 1990; Mellah et al. 2000; Eloseily et al. 2002) and the Random Finite Element Method (RFEM) (Fenton and Grifith, 2008). The RFEM involves generating a random field of soil properties with controlled mean, standard deviation and spatial correlation length, which is then mapped onto a finite element mesh.

In the past, SFEM has been developed using different expansions of stochastic variables. In this paper, we present SFEM using the method of Generalized Polynomial Chaos (GPC). In the first part of the paper, a new algorithm based on RFEM using the Circulant Embedding method (Lord et al. 2014) is presented in order to generate the random fields. In the second part, development of SFEM based on the Karhunen-Loeve
Expansion for stochastic process discretisation and GPC is described. Finally, in the last part of the paper, the problem of settlements of a foundation is solved by the two methods and the results are compared.

\section{PROBLEM DESCRIPTION AND MODEL FORMULATION}

Let us consider a general boundary value problem of computation of probable deformation of a body of arbitrary shape having randomly varying material properties caused by the application of a randomly varying load as shown in Fig. 1.

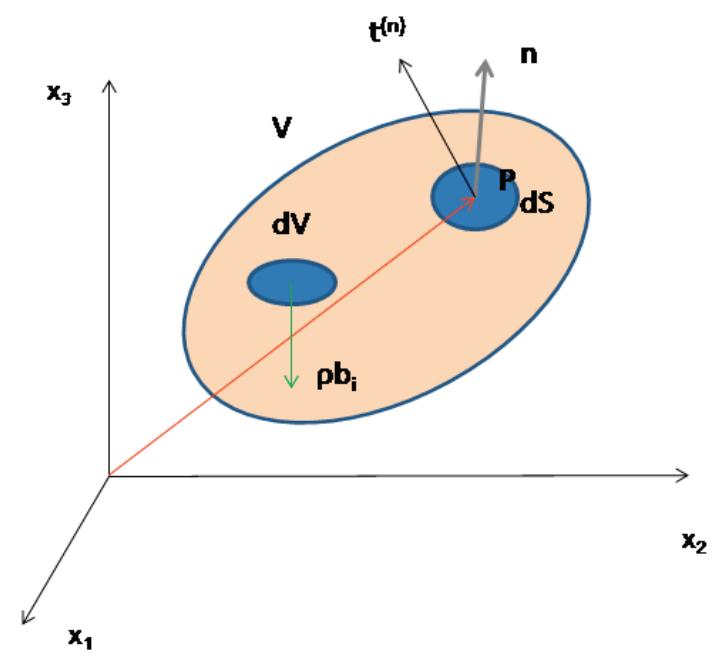

Fig. 1. Body of arbitrary shape 
According to the elasticity theory a boundary value problem can be described as follows

$$
\left\{\begin{array}{l}
\sigma_{i j, j}(\boldsymbol{x}, \boldsymbol{\omega})=f(\boldsymbol{x}, \boldsymbol{\omega}) \text { in } D \times \Omega, \\
\sigma_{i j}(\boldsymbol{x}, \boldsymbol{\omega})=C_{i j k l}(\boldsymbol{x}, \boldsymbol{\omega}) \varepsilon_{k l}(\boldsymbol{x}, \boldsymbol{\omega}) \text { in } D \times \Omega, \\
u(\boldsymbol{x}, \boldsymbol{\omega})=g_{D} \text { in } B_{D}, \\
\sigma_{i j}(\boldsymbol{x}, \boldsymbol{\omega}) n_{j}=g_{N} \text { in } B_{D} .
\end{array}\right.
$$

An in the weak form as

$$
a(u, v)=l(v)
$$

where

$$
\begin{gathered}
a(u, v)=\int_{D} \varepsilon^{T}(v) C(\boldsymbol{x}, \boldsymbol{\omega}) \varepsilon(u) d x, \\
l(v)=\int_{D} f(\boldsymbol{x}, \boldsymbol{\omega}) \cdot v d x+\int_{B_{N}} g_{N} \cdot v d x \\
-\int_{B_{D}} \varepsilon^{T}(v) C(\boldsymbol{x}, \boldsymbol{\omega}) \varepsilon(u) d x \cdot g_{D} .
\end{gathered}
$$

In the case of homogenous boundaries conditions the test function and the operators are determined as follows

$$
\begin{aligned}
& v \in V^{h} \in L^{2}\left(\Omega, L^{2}(D)\right), \\
& a: V^{h} \times V^{h} \rightarrow \mathbb{R}, \\
& l: V^{h} \rightarrow \mathbb{R} .
\end{aligned}
$$

In essence the solution of the problem is a function of the form $u \in \Omega \times D \rightarrow \mathbb{R}$, i.e., a random field and is not a deterministic function.

Although the procedure presented in the following sections is general and applicable to any boundary value problem, a specific problem of a plane-strain strip load on a semi-infinite elastic foundation with elastic modulus $(E)$ varying randomly in space is considered for simplicity of illustration (Fig. 2). The foundation loading in general form is not specified and also can vary randomly. In order to model the problem assuming the sample space $(\Omega, \mathcal{F}, \mathbb{P})$ where $\mathcal{F}$ is the $\sigma$-algebra and is considered to contain all the information that is available, $\mathbb{P}$ is the probability measure and the spatial domain of the soil is $\subset \mathbb{R}^{2}$. The elasticity modulus $\{E(\boldsymbol{x}, \boldsymbol{\omega}): \in D \times \Omega\}$ and the foundation load $\{f(\boldsymbol{x}, \boldsymbol{\omega}): \in D \times \Omega\}$ considered as second order random fields and their functions are determined $E, f: D \times \Omega \rightarrow \mathbb{R} \in V=L^{2}\left(\Omega, L^{2},(D)\right)$ and characterized by specific distribution, in our case as Gaussians. The expected value of a quantity of the problem is given by the following norm

$$
\|\cdot\|_{L^{2}\left(\Omega, L^{2}(D)\right)}=\int_{\Omega D} \int_{D}|\cdot|^{2}(x, \omega) d x d \mathbb{P}=\mathbb{E}\left(\|\cdot\|_{L^{2}(D)}\right)<\infty
$$

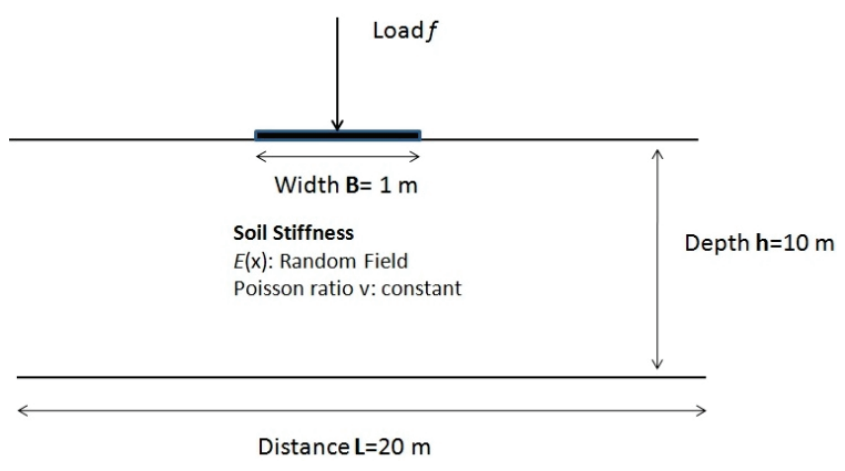

Fig. 2. Plane-strain strip load on a semi-infinite elastic foundation

\subsection{RANDOM FINITE ELEMENT}

The most common way to solve this problem is to create a random field of the soil properties which is mapped to a grid of finite element and then for different every time realization of the fields $\{E(\cdot, \omega)\}$ to solve an ordinary boundary value problem using the Monte Carlo method.

The problem of single and multiple footings has previously been studied using RFEM analysis by Paice et al. 1996 and Fenton and Griffiths 2002, 2005. In the work of Fenton and Griffiths, the results obtained in a probabilistic settlement analysis using a stochastic finite element method based on first order second moment approximations are compared with the random finite element method based on generation of random fields combined with Monte Carlo simulations. One of the main characteristics of these problems is how to create random fields. A majority of the researchers in the past followed the method of Local Average Subdivision (LAS) (Fenton and Vanmarcke 1990). In the current work, the random field is generated by the Circulant Embedding method (Lord et al. 2014) using the Fast Fourier Algorithm resulting in, as will be shown in the following sections, an exact simulation of stochastic processes.

Following the random field generation the displacement field $u_{k}(x)$ for each realization takes place. At the end of all running the statistical moment based on the Monte Carlo method is calculated. The problem for each realization $E_{\kappa}(\cdot, \omega)$ in the weak form is

$$
\alpha_{\kappa}(u, v)=l(v) .
$$

And the expected values at the end

$$
\mathbb{E}(u(\boldsymbol{x}))=\frac{1}{K} \sum_{k=1}^{K} u_{k}(\boldsymbol{x}) .
$$


And the variance

$$
\operatorname{Var}(u(\boldsymbol{x}))=\frac{1}{K-1} \sum_{k=1}^{K}\left(u_{k}(\boldsymbol{x})-\mathbb{E}(u(\boldsymbol{x}))^{2} .\right.
$$

\subsection{RANDOM FIELD GENERATION}

The Circulant Embedding method (Lord et al. 2014) is a technique used for the generation of realizations of Gaussian stochastic processes. This technique has two main advantages among others. The first is that the statistical properties of the generated process are exactly the same process that we aim. The second advantage arises from the Fast Fourier Transform Algorithm which significantly reduces the computational cost. This method seems to have initially been studied in problems of one dimension by Davies and Harte (1987) and more systematically by Dembo et al. (1989), Dietrich and Newsam (1993, 1997), Gneiting (2000), Stein (2001), Craigmile (2003), and Percival (2006). Extension of the method to multi-parameter problems was studied by Wood (1999), Helgasonetal (2011) whereas in random fields by Dietrich and Newsam (1993), Wood and Chan
(1994, 1997), Stein (2002, 2012), Gneiting et al. (2006). Considerable work was recently featured by Lord et al. (2014) whose principles are followed in this work. According to the method, in the case where the samples are uniformly distributed in space in a two-dimensional problem, then the covariance matrix $C$ is Toeplitz (Appendix A) and has as elements Toeplitz blocks (block Toeplitz with Toeplitz blocks (BTTB)). The covariance matrix can be described by the Fast Fourier Algorithm

$$
C=F D F^{H}
$$

where

$$
F_{N=n_{1} n_{2} \times n_{1} n_{2}}=F_{1} \otimes F_{2} .
$$

The $F_{1}, F_{2}$ are the Fourier matrices with dimensions of $n_{1} \times n_{1}$ and $n_{1} \times n_{2}$, respectively, and the diagonal matrix $D$ includes the eingenvalues of the covariance matrix. For the application of the method the covariance matrix has to be circulant and for that reason using the BTTB matrix a new circulant (Appendix A) matrix is created with $n_{2}$ blocks of circulant matrices $n_{1} \times n_{1}$ which is represented uniquely by the reduced matrix $\boldsymbol{C}_{\text {red }}=\left[\mathbf{c}_{0}, \ldots, \mathbf{c}_{n_{2}-1}\right]$. The latter can be replaced uniquely by the vector $\boldsymbol{c}_{\text {red }} \in \mathbb{R}^{n_{1} n_{2}}$.

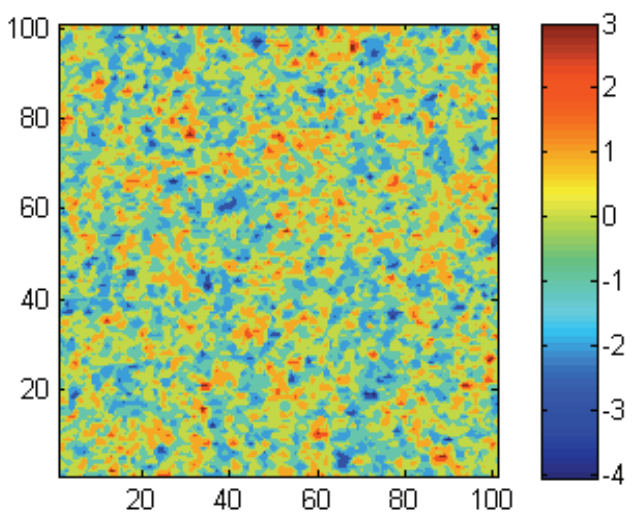

Fig. 3. Random field with dimension $D=[0.100] \times[0.100]$ and correlation length $\lambda_{x}=\lambda_{y}=\frac{1}{10}$
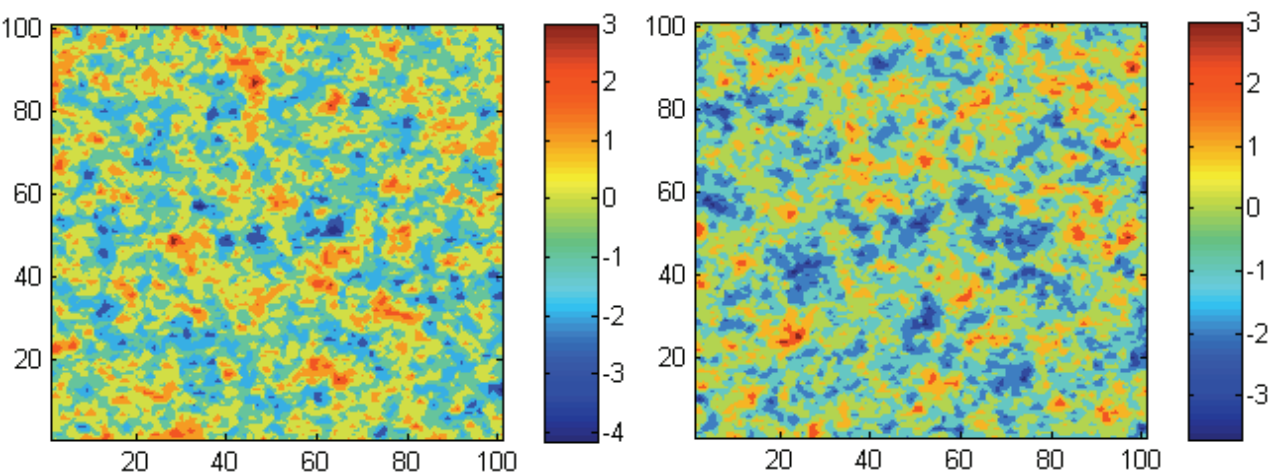

Fig. 4. Random field with dimension $D=[0.100] \times[0.100]$ and correlation length $\lambda_{x}=\lambda_{y}=\frac{2}{10}$ 

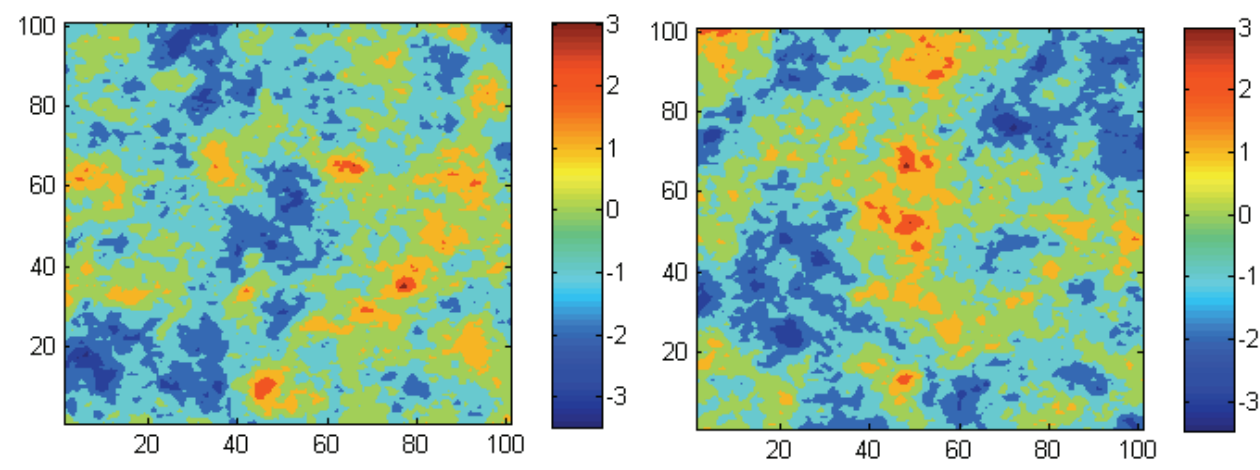

Fig. 5. Random field with dimension $D=[0.100] \times[0.100]$ and correlation length $\lambda_{x}=\lambda_{y}=1$
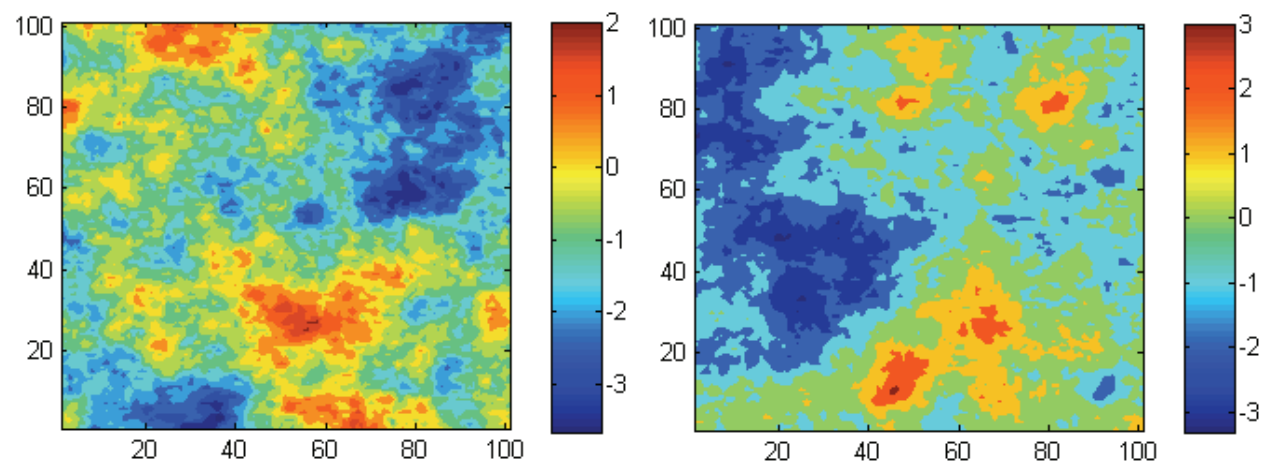

Fig. 6. Random field with dimension $D=[0.100] \times[0.100]$ and correlation length $\lambda_{x}=\lambda_{y}=2$

Following the process described and provided that the covariance matrix has non-negative and real eigenvalues we get

$$
Z=D^{1 / 2} \xi \mu \varepsilon \xi \sim N\left(0.2 I_{N}\right) .
$$

And finally,

$$
Z=X+i Y
$$

where

$$
\begin{aligned}
& X \sim N(0, C), \\
& Y \sim N(0, C) .
\end{aligned}
$$

Considering the covariance matrix

$$
C(x, y)=\sigma^{2} \exp \left(-\frac{\left|x_{j}-x_{i}\right|}{\lambda_{x}}-\frac{\left|y_{j}-y_{i}\right|}{\lambda_{y}}\right) .
$$

In Figs. 3 to 6, examples of random fields realization for different correlation lengths of the above covariance matrix are presented.

\section{THE STOCHASTIC FINITE ELEMENT METHOD (SFEM)}

The SFEMs have a wide range of applications and are used to solve problems in various branches of science. In the following paragraphs we introduce the procedure to solve problems in geotechnical engineering using the Stochastic Finite Element Method based on Generalized Polynomial Chaos.

\subsection{KARHUNEN-LOEVE EXPANSION}

One of the major points of the SFEM is the separation of deterministic part from the stochastic part of the formulation. Thus the method has two types of discretization, the ordinary FEM discretization of geometry and the stochastic discretization of random fields. In the current paper, in order to reach in these results the Karhunen-Loeve expansion has been used which is the most efficient method for the discretization of a random field, requiring the smallest number of random variables to represent the field within a given level of accuracy. Based on that the stochastic process of Young's modulus over the spatial domain with a known mean value $\widetilde{E}(x)$ and covariance matrix $\operatorname{Cov}\left(x_{1}, x_{2}\right)$ is given by

$$
E(\boldsymbol{x}, \xi(\omega))=\widetilde{E}(\boldsymbol{x})+\sum_{\kappa}^{\infty} \sqrt{\lambda_{\kappa}} \varphi_{\kappa}(\boldsymbol{x}) \xi_{\kappa}(\boldsymbol{\omega}) .
$$

In practice, calculations were carried out over a finite number of summations (for example, 1-5), so the approximate stochastic representation is given by the trancuated part of expansion 


$$
E(\boldsymbol{x}, \xi(\omega))=\widetilde{E}(x)+\sum_{\kappa=1}^{K} \sqrt{\lambda_{\kappa}} \varphi_{\kappa} \xi_{\kappa}(\omega)
$$

where

$\lambda_{\kappa}$ are the eingenvalues of the covariance function,

$\varphi_{k}(\boldsymbol{x})$ are the eingenfunctions of the covariance function $\operatorname{Cov}\left(x_{1}, x_{2}\right)$,

$$
\begin{aligned}
& x \in D \text { and } \omega \in \Omega, \\
& \xi=\left[\xi_{1}, \xi_{1}, \ldots, \xi_{M}\right]: \Omega \rightarrow \Gamma \subset \mathbb{R}^{M} \text { and } \\
& \Gamma=\Gamma_{1} \times \Gamma_{1} \times \ldots \times \Gamma_{M} .
\end{aligned}
$$

The pairs of eingenvalues and eingenfunctions were obtained by the equation

$$
\int_{D} C\left(x_{1}, x_{2}\right) \varphi_{\kappa}\left(x_{2}\right)=\lambda_{\kappa} \varphi_{\kappa}\left(x_{1}\right) .
$$

\subsection{GALERKIN APPROXIMATION}

The Karhunen-Loève expansion method enables one to replace the calculating procedure for the expected value using instead of the abstract space $\Omega$ of random fields $\xi$ their figures and finally to solve a deterministic problem in space $D \times \Gamma \subset \mathbb{R}^{M}$ instead of space $D \times \Omega$. By performing such replacements, in fact, a deterministic problem is solved, in contrast to the case of Monte Carlo where a large number of problems is carried out. According that the test function of the weak form determined by $v \in L_{p}^{2}\left(\Gamma, H_{0}^{1}(D)\right)$ while the solution of the problems in the general form of the boundaries conditions is a function $\tilde{u} \in W=L_{p}^{2}\left(\Gamma, H_{g}^{1}(D)\right)$ which is satisfied by the equation

$$
\widetilde{\alpha}(\widetilde{u}, v)=l(v) \forall v \in L_{p}^{2}\left(\Gamma, H_{0}^{1}(D)\right) .
$$

And

$$
\begin{aligned}
& \tilde{\alpha}: W \times V \rightarrow \mathbb{R}, \\
& l: V \rightarrow \mathbb{R}, \\
& \tilde{\alpha}(\tilde{u}, v)=\int_{\Gamma} \rho(\boldsymbol{y}) \int_{D} \varepsilon^{T}(v) C(\boldsymbol{x}, \boldsymbol{y}) \varepsilon(u) d x d y .
\end{aligned}
$$

In the general case, where the load presents randomness

$$
\begin{aligned}
\tilde{l}(v) & =\int_{\Gamma} \rho(\boldsymbol{y})\left[\int_{D} f(\boldsymbol{x}, \boldsymbol{\omega}) \cdot v d x+\int_{B_{N}} g_{N} \cdot v d x\right. \\
& \left.-\int_{B_{D}} \varepsilon^{T}(v) C(\boldsymbol{x}, \boldsymbol{\omega}) \varepsilon(u) d x \cdot g_{D}\right] d y
\end{aligned}
$$

where $\rho: \Gamma \subset \mathbb{R}$ is the $\eta$ joint density of independent random variables $\xi$.

In order to solve the problem according to the finite element method in the current paper we consider a triangle $K$ with nodes $N_{i}\left(x^{(i)}, y^{(i)}\right), i=1,2,3$. To each node $N_{i}$ there is a hat function $\varphi_{i}$ associated, which takes the value 1 at node $N_{i}$ and 0 at the other two nodes. Each hat function is a linear function on $K$ so it has the form

$$
\varphi_{i}=a_{i}+b_{i} x+c_{i} y .
$$

The test $v$ function belongs to the space

$$
V^{h}=\operatorname{span}\left\{\varphi_{1}, \varphi_{2}, \ldots, \varphi_{N}\right\} \subset H_{0}^{1}(D)
$$

Any type of higher order shape functions can be used although it will increase the computational cost.

In order to solve problem 1 we have to create the new space $L_{p}^{2}\left(\Gamma, H_{0}^{1}(D)\right)$. For that reason the subspace $S^{k} \subset L_{p}^{2}(\Gamma)$ is considered as (Lord et al. 2014)

$$
S^{k}=\operatorname{span}\left\{\psi_{1}, \psi_{2}, \ldots, \psi_{\kappa}\right\} .
$$

Using the dyadic product of the space $V^{h}, S^{k}$ the space $L_{p}^{2}\left(\Gamma, H_{0}^{1}(D)\right)$ is created. Thus,

$$
V^{h k}=V^{h} \otimes V^{k}=\operatorname{span}\left\{\varphi_{i} \psi_{j}, i=1 \ldots N, j=1, \ldots, Q\right\}
$$

The space $V^{h k}$ has dimension $Q N$ and regards the test function $v$. In the case where there exists $N_{B}$ finite element supported by boundaries condition then the subspace of solution is

$$
W^{h k}=V^{h} \oplus \operatorname{span}\left\{\varphi_{N+1}, \varphi_{N+2}, \ldots, \varphi_{N+N B}\right\}
$$

\subsection{GENERALIZED POLYNOMIAL OF CHAOS AND STOCHASTIC GALERKIN SOLUTION}

Assuming that the $S_{i}^{k}$ represents a space of univariate orthonormal polynomial of variable $y_{i} \subset \Gamma_{l} \subset \mathbb{R}$ with order $k$ or lower,

$$
S_{i}^{k}=\left\{P_{a_{i}}^{i}\left(y_{i}\right), a_{i}=1,2, \ldots, k\right\}, \quad i=1, \ldots, M .
$$

The tensor product of the $M S_{i}^{k}$ subspace results in the space of the Generalized Polynomial Chaos

$$
S^{k}=S_{1} \otimes S_{2} \ldots \otimes S_{M} .
$$

Xiu and Karniadakis (2003) show the application of the method for different kinds of orthonormal poly- 
nomials and in the current paper the Hermite polynomial was used with the following characteristics

$$
\begin{gathered}
P_{0}=1, \quad\left\langle P_{i}\right\rangle=0, \quad i>0, \\
\left\langle P_{m} P_{n}\right\rangle=\int_{\Gamma} P_{m}(\boldsymbol{y}) P_{n}(\boldsymbol{y}) \rho(\boldsymbol{y}) d y=\gamma_{n} \delta_{m n},
\end{gathered}
$$

where

$\gamma_{n}=\left\langle P_{n}^{2}\right\rangle$ are the normalization factors,

$\delta_{m n}$ is the Kronecker delta,

$$
\rho(y)=\frac{1}{\sqrt{2 \pi}} e^{-\frac{x}{2}}
$$

is the density function.

And

$$
P_{n}=(-1)^{n} e^{\frac{x}{2}} \frac{d^{n}}{d x^{n}} e^{-\frac{x}{2}} .
$$

The function $u \in W^{h k}$ can be written as the summation of $S^{k}$ polynomials base as

$$
u(\boldsymbol{x}, \boldsymbol{y})=\sum_{k=1}^{p} u_{k}(\boldsymbol{x}) \psi_{\kappa}(\boldsymbol{y}) .
$$

According to that and using the inner product of the weak form equation on each polynomial of the $S^{k}$ base we get

$$
\left.\left\langle a\left(\sum_{k=1}^{p} u_{k}(\boldsymbol{x}) \psi_{\kappa}(\boldsymbol{y}), v\right), \psi_{p}\right\rangle=<l(v), \psi_{p}\right\rangle
$$

The lhs of the equation can be written based on the solution's polynomial chaos expansion as

$$
\begin{gathered}
\left\langle a\left(\sum_{k=1}^{p} u_{k}(\boldsymbol{x}) \psi_{\kappa}(\boldsymbol{y}), v\right), \psi_{p}\right\rangle=\sum_{i=1}^{n d o f} \\
\sum_{k=1}^{p} u_{i k} \int_{\Gamma} \rho(\boldsymbol{y}) \psi_{\kappa}(\boldsymbol{y}) \psi_{p}(\boldsymbol{y}) \int_{D} B^{T} C(x, \boldsymbol{y}) B d x d y
\end{gathered}
$$

where $B$ is the strain displacement matrix.

Using the Karhunen-Loève expansion the stochastic elasticity tensor is given by

$$
C_{i j k l}(\boldsymbol{x}, \boldsymbol{y})=\widetilde{E}(\boldsymbol{x}) C_{i j k l}^{*}(\boldsymbol{x})+\sum_{m=1}^{M} \sqrt{\lambda_{m}} \varphi_{m} y_{m} C^{*}(\boldsymbol{x}) .
$$

$C_{i j k l}^{*}(\boldsymbol{x})$ is expressed in terms of (deterministic) Poisson's ratio as

$$
C_{i j k l}^{*}(\boldsymbol{x})=\frac{v}{(1+v)} \delta_{i j} \delta_{k l}+\frac{1}{2(1+v)}\left(\delta_{i k} \delta_{j l}+\delta_{l l} \delta_{j k}\right) .
$$

In the case of plane strain conditions

$$
C^{*}(\boldsymbol{x})=\frac{1}{1+v}\left[\begin{array}{ccc}
1-v & 0 & 0 \\
v & 1-v & 0 \\
0 & 0 & \frac{1-2 v}{v}
\end{array}\right] .
$$

From the above

$$
\begin{gathered}
\left\langle a\left(\sum_{k=1}^{p} u_{k}(\boldsymbol{x}) \psi_{\kappa}(\boldsymbol{y}), v\right), \psi_{p}\right\rangle \\
=\sum_{i=1}^{n d o f} \sum_{k=1}^{p} u_{i k} \int_{\Gamma} \rho(\boldsymbol{y}) \psi_{\kappa}(\boldsymbol{y}) \psi_{p}(\boldsymbol{y}) \int_{D} B^{T} \widetilde{E}(\boldsymbol{x}) C^{*}(\boldsymbol{x}) B d x d y \\
+\sum_{m=1}^{M} \sum_{i=1}^{n d o f} \sum_{k=1}^{p} u_{i k} \int_{\Gamma} \rho(\boldsymbol{y}) \psi_{\kappa}(\boldsymbol{y}) \psi_{p}(\boldsymbol{y}) y_{m} \\
\int_{D} \sqrt{\lambda_{m}} \varphi_{m} B^{T} C^{*}(\boldsymbol{x}) B d x d y .
\end{gathered}
$$

If we set

$$
\begin{gathered}
Q_{0}=\int_{\Gamma} \rho(\boldsymbol{y}) \psi_{\kappa}(\boldsymbol{y}) \psi_{p}(\boldsymbol{y}) d y, \\
Q_{m}=\int_{\Gamma} \rho(\boldsymbol{y}) \psi_{\kappa}(\boldsymbol{y}) \psi_{p}(\boldsymbol{y}) y_{m} d y, \\
K_{0}=\int_{D} B^{T} \widetilde{E}(\boldsymbol{x}) C^{*}(\boldsymbol{x}) B d x, \\
K_{m}=\int_{D} \sqrt{\lambda_{m}} \varphi_{m} B^{T} C^{*}(\boldsymbol{x}) B d x .
\end{gathered}
$$

The LHS of the weak form equation can be written as

$$
a(u, v)=Q_{0} \otimes K_{0}+\sum_{m=1}^{M} Q_{m} \otimes K_{m} .
$$

And the RHS of the weak form assuming constant load for simplicity

$$
\begin{gathered}
\left\langle l(v), \psi_{p}\right\rangle=\int_{\Gamma} \rho(\boldsymbol{y}) \psi_{p}\left[\int_{D} \varphi^{T} f(\boldsymbol{x}) d x+\int_{B_{N}} \varphi^{T} g_{N} d s\right. \\
\left.-\int_{B_{D}} B^{T}(v) C(\boldsymbol{x}, \boldsymbol{y}) B(u) d x \cdot g_{D}\right] d y .
\end{gathered}
$$


If we set

$$
\begin{gathered}
q_{0}=\int_{\Gamma} \rho(\boldsymbol{y}) \psi_{p}(\boldsymbol{y}) \psi_{1}(\boldsymbol{y}) d y, \\
q_{m}=\int_{\Gamma} \rho(\boldsymbol{y}) \psi_{p}(\boldsymbol{y}) \psi_{1}(\boldsymbol{y}) y_{m} d y, \\
K_{B_{0}}=\int_{B_{D}} B^{T} \widetilde{E}(\boldsymbol{x}) C^{*}(\boldsymbol{x}) B d x, \\
K_{B_{m}}=\int_{B_{D}} \sqrt{\lambda_{m}} \varphi_{m} B^{T} C^{*}(\boldsymbol{x}) B d x, \\
f_{0}=\int_{D} \varphi^{T} f(\boldsymbol{x}) d x, \\
t_{g N}=\int_{B_{N}} \varphi^{T} \cdot g_{N} d s .
\end{gathered}
$$

And

$$
\left\langle l(v), \psi_{p}\right\rangle=q_{0} \otimes\left(f_{0+} t_{g N}-K_{B 0} \cdot g_{d}\right)+\sum_{m=1}^{M} q_{m} \otimes K_{B m} .
$$

Finally, the system has the form

$$
K \cdot u=F, K \in \mathbb{R}^{\text {ndof } \cdot P \times \text { ndof } \cdot P} \text {, and } F \in \mathbb{R}^{\text {ndof } \cdot P} .
$$

The statistical moments of the displacement field arise by the properties of the Polynomial of Chaos expansion.
The expected value

$$
\begin{array}{r}
\mathbb{E}[u(\boldsymbol{x}, \boldsymbol{y})]=\mathbb{E}\left[\sum_{k=0}^{p} u_{k}(\boldsymbol{x}) \psi_{\kappa}(\boldsymbol{y})\right] \\
=\underbrace{u_{0}(\boldsymbol{x}) \mathbb{E}\left[\psi_{0}(\boldsymbol{y})\right]}_{1}+\underbrace{\sum_{k=1}^{p} u_{k}(\boldsymbol{x}) \mathbb{E}\left[\psi_{\kappa}(\boldsymbol{y})\right]}_{0}=u_{0}(\boldsymbol{x}) .
\end{array}
$$

And the variance

$$
\begin{gathered}
\sigma^{2}=\mathbb{E}(u(\boldsymbol{x}, \boldsymbol{y})-\mathbb{E}[u(\boldsymbol{x}, \boldsymbol{y})])^{2} \\
\left.=\mathbb{E}\left(\sum_{k=0}^{p} u_{k}(\boldsymbol{x}) \mathbb{E}\left[\psi_{\kappa}(\boldsymbol{y})\right)\right]-u_{0}(\boldsymbol{x})\right) \Rightarrow \\
\sigma^{2}=\sum_{k=0}^{p} u_{k}^{2}(\boldsymbol{x}) \mathbb{E}\left[\psi_{\kappa}^{2}\right] .
\end{gathered}
$$

\section{NUMERICAL EXAMPLE}

The application of the numerical algorithms described in the previous paragraphs is presented and results are compared to those obtained from RFEM using Monte Carlo simulations. The problem shown in Fig. 2 is solved and the geometry of the finite elements used is presented in Fig. 7. The input data of the problem is the random field modulus with a constant average value equal to $100 \mathrm{MPa}$ and a fixed Poisson ratio equal to 0.3 . Calculations have been made for ten different coefficients $v_{e}=\frac{\sigma_{E}}{\mu_{E}}$ of the elastic modulus with a minimum value of 0.1 and then with step 0.1 to a maximum value equal to 1 . This problem was solved for four different correlation

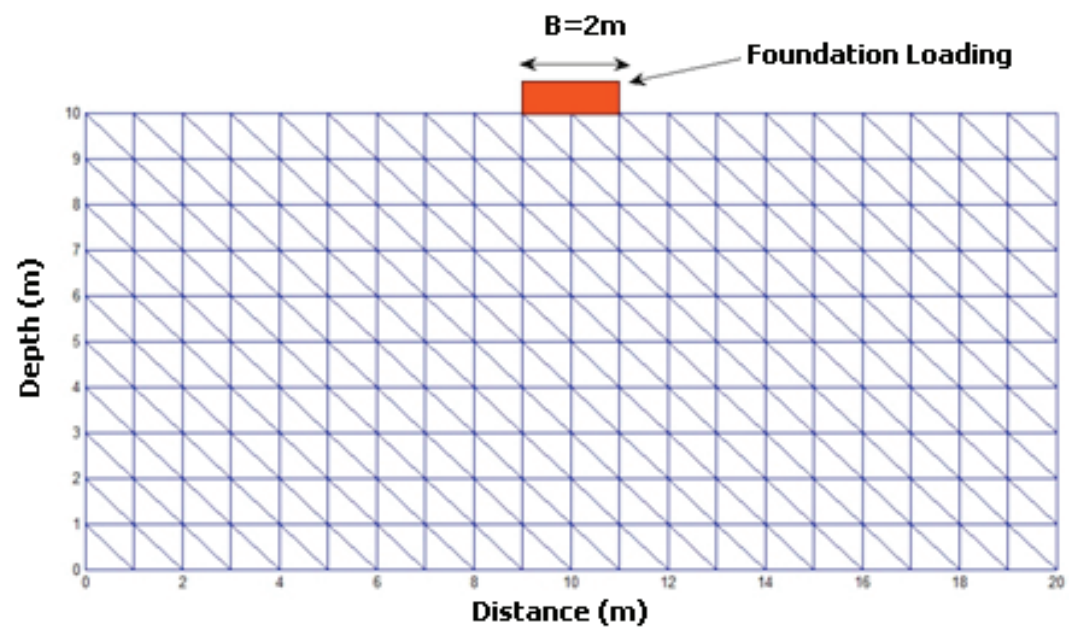

Fig. 7. Finite element mesh 
lengths $\lambda_{x}=\lambda_{y}$ the $0.1,0.5,1,2$. For the RFEM 100 simulations are executed creating 200 realisation (Fig. 8) while for SFEM there were used one dimensional Hermite GPC with order 5 (Xiu and Karniadakis 2003). In the figure, the results of settlement are shown, while in Figs. 9-13 comparatively the results of the expected values of the two methods are presented. It is observed that the results show slight differences up to a value of $v_{e}=0.5$, while the continuous increase of $v_{e}$ leads to the maximum value of difference when $v_{e}$ is equal to 1 . The same effect on the results presents the increasing of the correlation length. In practice, it can be considered an upper limit of variability for many soils (e.g., Lee et al. 1983) for elasticity with $v_{e}=0.5$. Figure 14 shows the results for $v_{e}=0.5$ with different values of correlation lengths being presented. In this case the differences do not exceed 2.4\%. In Appendix B, a comparison of the results for the case of $v_{e}=0.5$ and $\lambda_{x}=\lambda_{y}=2$ is presented.
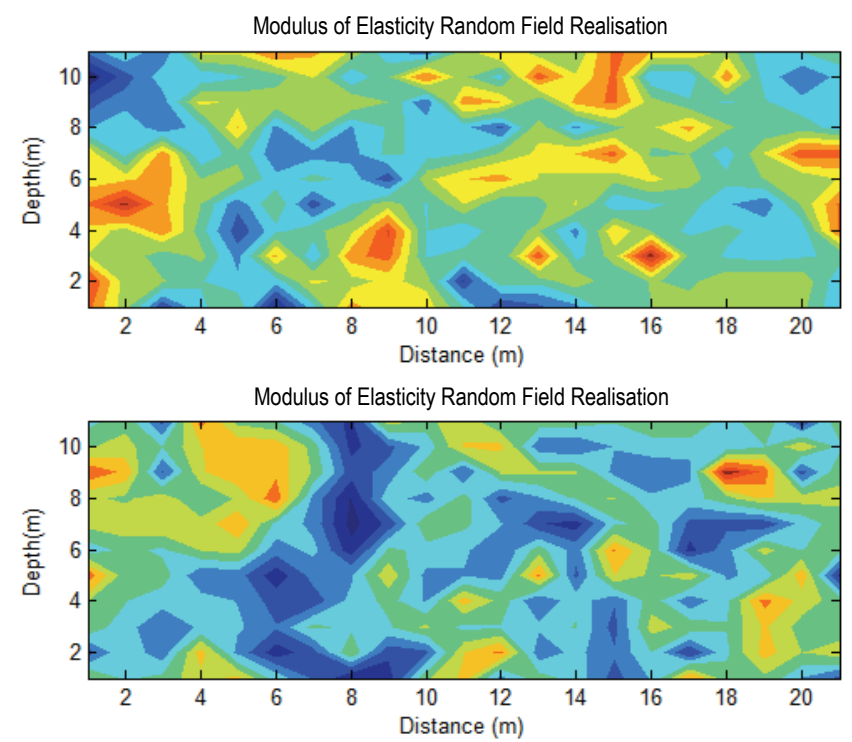

Fig. 8. Modulus of Elasticity Random field realization

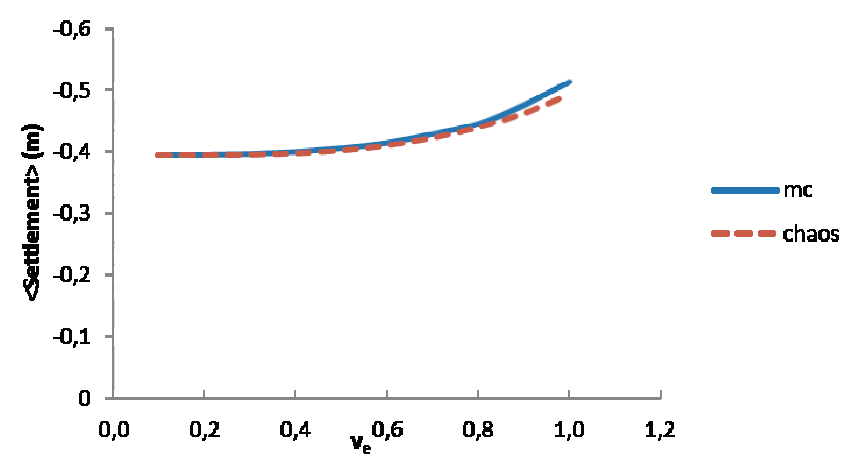

Fig. 9. Expected vertical displacement for correlation length $\lambda_{x}=\lambda_{y}=0.1$ and for various values of $v_{e}$

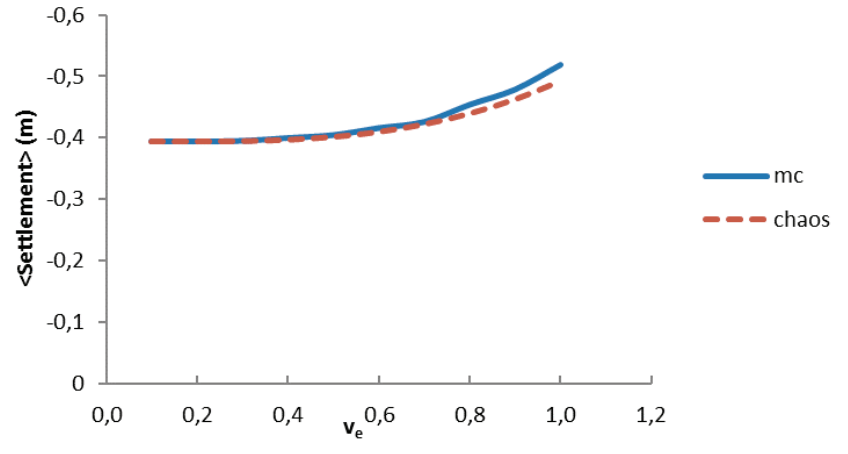

Fig. 10. Expected vertical displacement for correlation length $\lambda_{x}=\lambda_{y}=0.5$ for various values of $v_{e}$

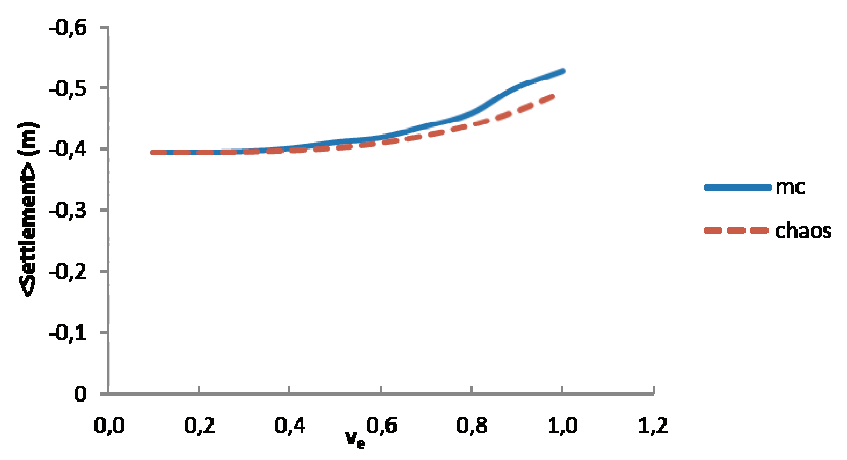

Fig. 11. Expected vertical displacement for correlation length $\lambda_{x}=\lambda_{y}=1$ for various values of $v_{e}$

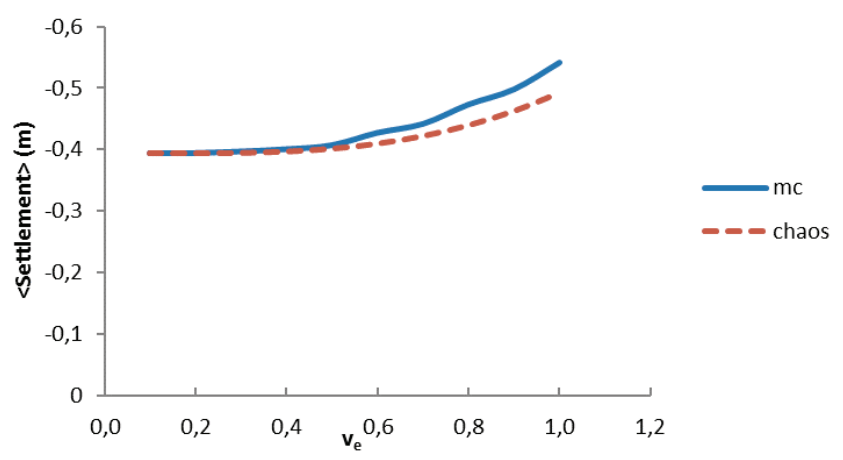

Fig. 12. Expected vertical displacement for correlation length $\lambda_{x}=\lambda_{y}=2$ for various values of $v_{e}$

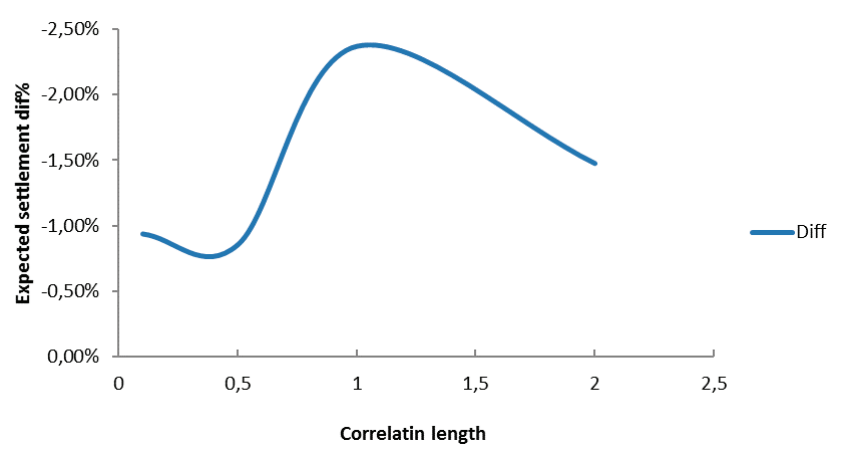

Fig. 13. Settlement differences for different correlation's lengths and $v_{e}=0.5$ 


\section{CONCLUSIONS}

A procedure of conducting a Stochastic Finite Element Analysis of geotechnical structures where uncertainty arises due to spatial variability of mechanical parameters of soil/rock has been presented. Two different approaches in order to quantify uncertainty are discussed. The first approach involves generating a random field based on Circulant embedding method and the second Stochastic Finite Element using Polynomial Chaos. An analysis of settlement of a plane strain strip load on an elastic foundation has been given as an example of the proposed approach. It is shown that the results of SFEM using polynomial chaos compare well with those obtained from Random Finite Element Method. The main advantage of using the proposed methodology is that a large number of realisations which have to be made for RFEM are avoided, thus making the procedure viable for realistic practical problems.

\section{APPENDIX A}

\section{Definition 1}

A Toeplitz matrix is an $n \times n$ matrix $T_{n}=\left[t_{k, j}: k, j=0\right.$, $1, \ldots, n-1]$ where $t_{k, j}=t_{k-j}$, i.e., a matrix of the form

$$
T_{n}=\left[\begin{array}{ccccc}
t_{0} & t_{-1} & t_{-1} & \ldots & t_{-(n-1)} \\
t_{1} & t_{0} & t_{-1} & & \vdots \\
t_{2} & t_{1} & t_{0} & & \\
\vdots & & & \ddots & \\
t_{n-1} & & & \ldots & t_{0}
\end{array}\right] .
$$

\section{Definition 2}

When every row of the matrix is a right cyclic shift of the row above it so that $t_{k}=t_{-(n-k)}$ for $k=1,2$, $\ldots, n-1$. In this case, the matrix is called Circulant and is equal to

$$
C_{n}=\left[\begin{array}{ccccc}
t_{0} & t_{-1} & t_{-2} & \ldots & t_{-(n-1)} \\
t_{-(n-1)} & t_{0} & t_{-1} & & \\
t_{-(n-2)} & t_{-(n-1)} & t_{0} & & \vdots \\
& & & \ddots & \\
t_{-1} & t_{-2} & & \ldots & t_{0}
\end{array}\right] .
$$

\section{Definition 3}

If $C_{k}$ is $n_{1} \times n_{1}$ Toeplitz matrix, then the $N \times N$ matrix with $N=n_{1} n_{2}$ and the form

$$
C=\left[\begin{array}{ccccc}
C_{0} & C_{-1} & \ldots & C_{2-n_{2}} & C_{1-n_{2}} \\
C_{1} & C_{0} & C_{-1} & \ddots & C_{2-n_{2}} \\
\vdots & \ddots & \ddots & & \vdots \\
C_{n_{2}-2} & \ddots & C_{1} & C_{0} & C_{-1} \\
C_{n_{2}-1} & C_{n_{2}-1} & \ldots & C_{1} & C_{0}
\end{array}\right]
$$

is called Block Toeplitz matrix with Toeplitz Blocks (BTTB).

\section{APPENDIX B}

Comparison of results for the case of $v_{e}=0.5$ and $\lambda_{x}=\lambda_{y}=2$.
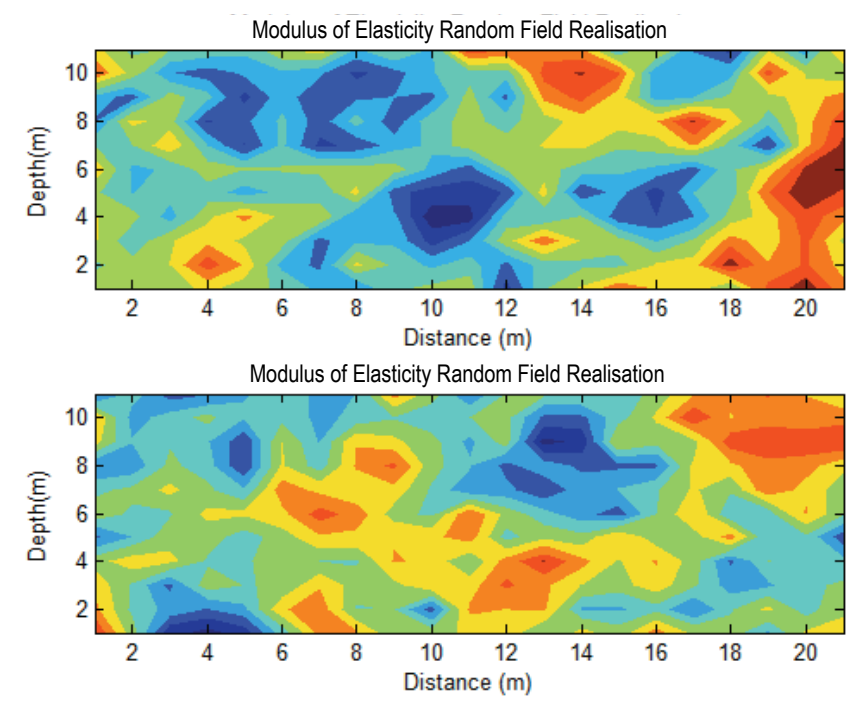

Fig. A1. Realization of Modulus of Elasticity for the case of $v_{e}=0.5$ and $\lambda_{x}=\lambda_{y}=2$

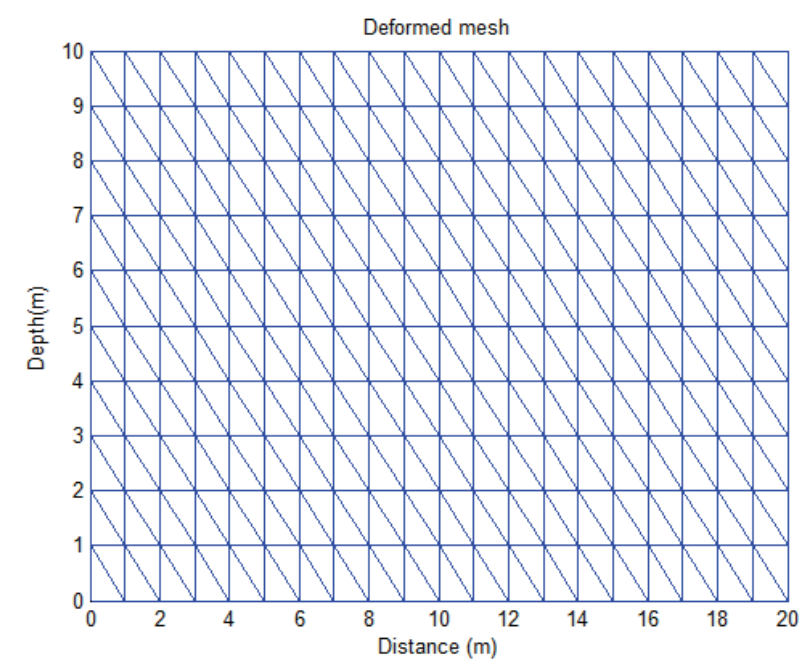

Fig. A2. Finite element mesh 


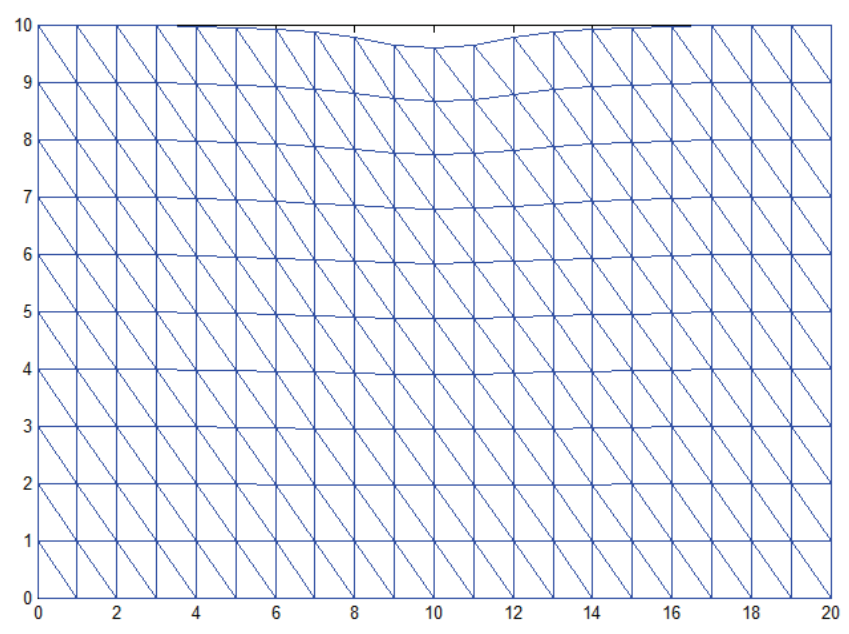

Fig. A3. Deformed mesh
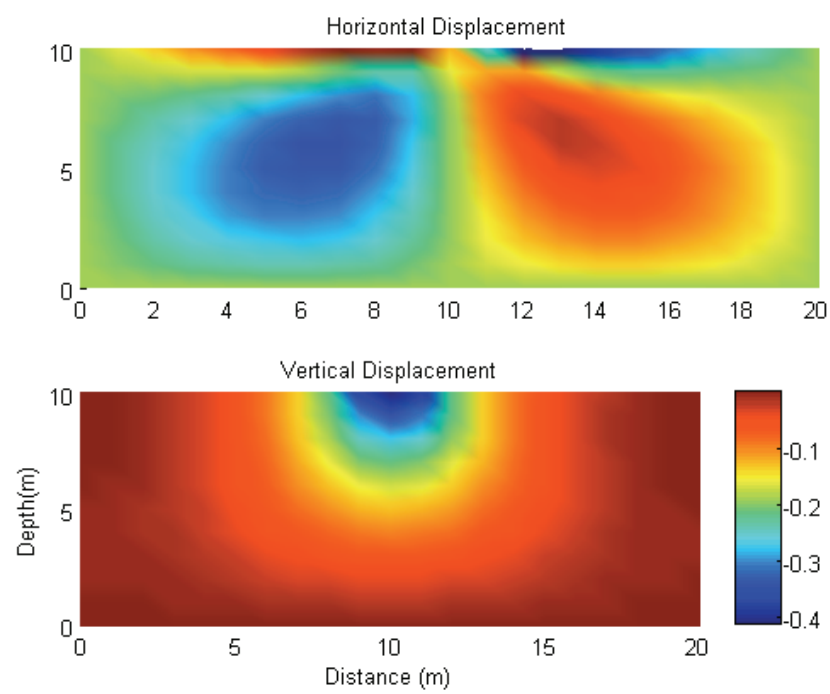

Fig. A4. Displacement field results of gPC method for the case of $v_{e}=0.5$ and $\lambda_{x}=\lambda_{y}=2$
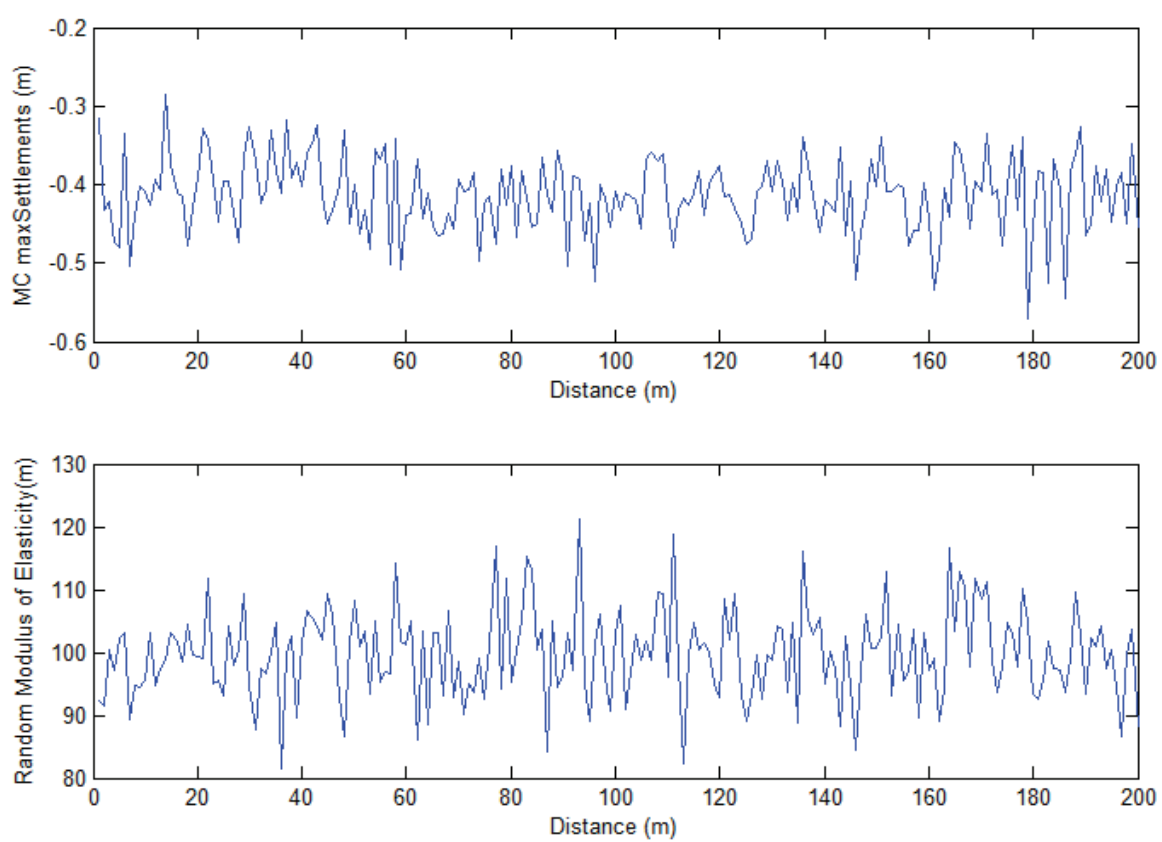

Fig. A5. Settlements and Modulus of Elasticity realizations beneath foundation (Monte Carlo Method) for the case of $v_{e}=0.5$ and $\lambda_{x}=\lambda_{y}=2$

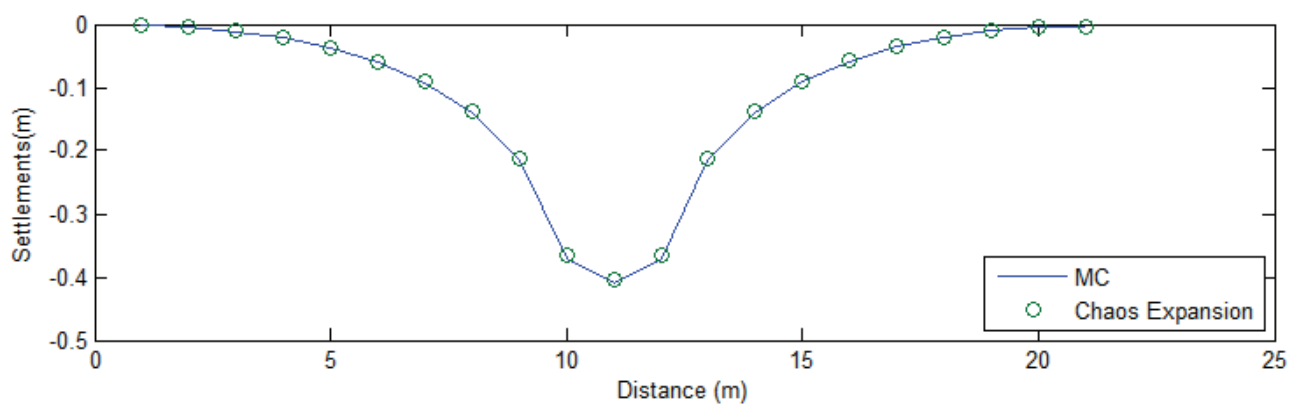

Fig. A6. Comparison of settlement results for the two methods for the case of $v_{e}=0.5$ and $\lambda_{x}=\lambda_{y}=2$ 

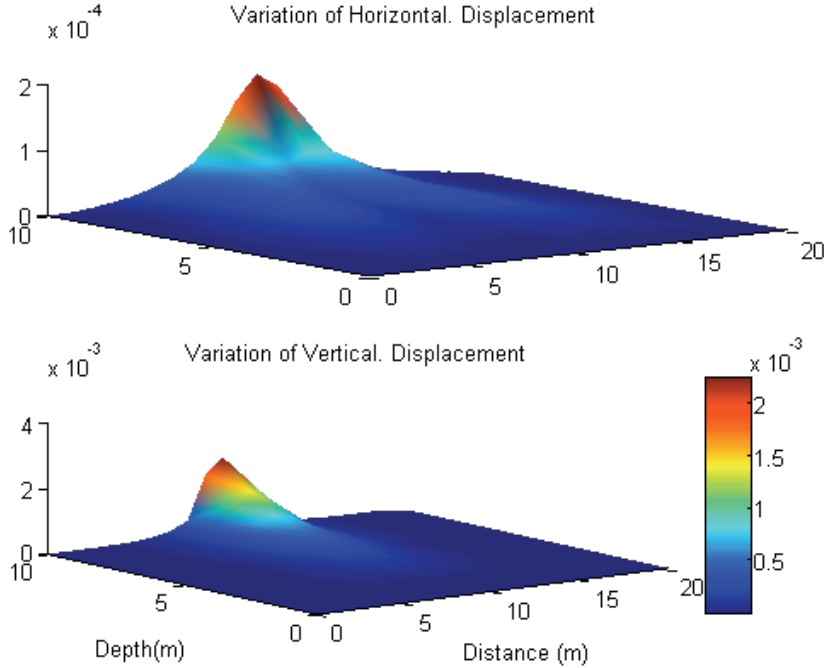

Fig. A7. Variation of displacement Monte Carlo results for the case of $v_{e}=0.5$ and $\lambda_{x}=\lambda_{y}=2$

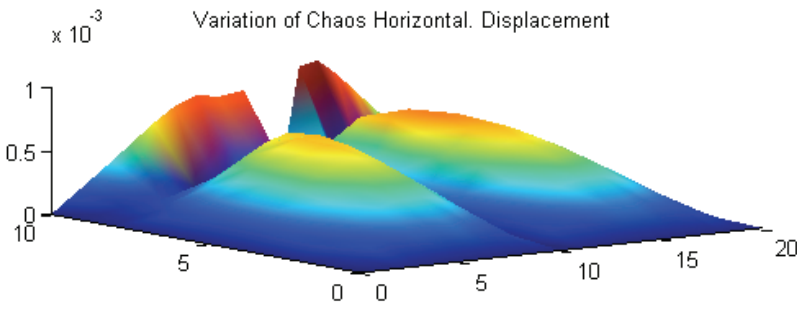

Variation of Chaos Vertical. Displacement

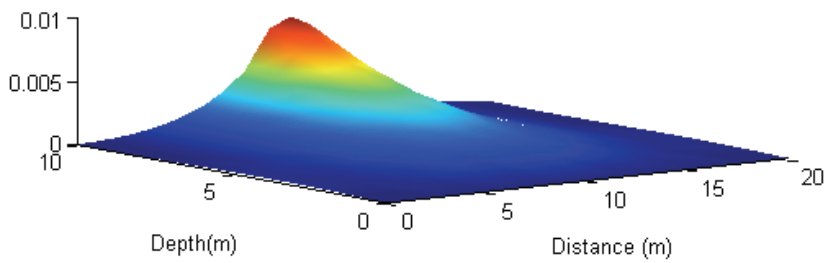

Fig. A8. Variation of displacement gPC results for the case of $v_{e}=0.5$ and $\lambda_{x}=\lambda_{y}=2$

\section{REFERENCES}

[1] Davies R., HaRte D., Tests for Hurst effect, Biometrika, 1987, 74(4), 95-101.

[2] Dembo A., Mallows C., Shepp L., Embedding nonnegative definite Toeplitz matrices in nonnegative definite circulant matrices, with applications to covariance estimation, IEEE Transactions on Information Theory, 1989, $35,1206-1212$.
[3] Dietrich C., Newsam G., A fast and exact simulation for multidimensional Gaussian stochastic simulations, Water Resources Research, 1993, 29(8), 2861-2869.

[4] Dietrich C., Newsam G., Fast and exact simulation of stationary Gaussian processes through circulant embedding of the covariance matrix, SIAM Journal on Scientific Computing, 1997, 18(4), 1088-1107.

[5] Gneiting T., Power-law correlations, related models for long-range dependence and their simulation, Journal of Applied Probability, 2000, 37(4), 1104-1109.

[6] Gneiting T., Sevčíková H., Percival D., Schlather M., JIANG Y., Fast and exact simulation of large Gaussian lattice systems in R2: Exploring the limits, Journal of Computational and Graphical Statistics, 2006, 15(3), 483-501.

[7] STEIN M., Local stationarity and simulation of self-affine intrinsic random functions, IEEE Transactions on Information Theory, 2001, 47(4), 1385-1390.

[8] STEIn M., Fast and exact simulation of fractional Brownian surfaces, Journal of Computational and Graphical Statistics, 2002, 11(3), 587-599.

[9] Stein M., Simulation of Gaussian random fields with one derivative, Journal of Computational and Graphical Statistics, 2012, 21(1), 155-173.

[10] Wood A., Chan G., Simulation of stationary Gaussian processes in $[0,1]^{d}$, Journal of Computational and Graphical Statistics, 1994, 3(4), 409-432.

[11] Paice G.M., Griffiths D.V., Fenton G.A., Finite element modeling of settlements on spatially random soil, J. Geotech. Eng., 1996, 122(9), 777-779. Smith, I. M., and Griffiths,

[12] Fenton G.A., GRIFFITHS D.V., Statistics of block conductivity through a simple bounded stochastic medium, Water Resour. Res., 1993, 29(6), 1825-1830.

[13] Fenton G.A., Griffiths D.V., Probabilistic foundation settlement on spatially random soil, J. Geotech. Geoenviron. Eng., 2002, 128(5), 381-390.

[14] FENTON G.A., GRIFFITHS D.V., Three-dimensional probabilistic foundation settlement, J. Geotech. Geoenviron. Eng., 2005, 131(2), 232-239.

[15] Fenton G.A., Griffiths D.V., Risk assessment in geotechnical engineering, Wiley, Hoboken, N.J. 2008.

[16] Fenton G.A., Vanmarcke E.H., Simulation of random fields via local average subdivision, J. Eng. Mech., 1990, 116(8), 1733-1749.

[17] Ghanem R.G., Spanos P.D., Stochastic finite elements: A spectral approach, Springer-Verlag, New York 1991.

[18] Griffiths D.V., Fenton G.A., Seepage beneath water retaining structures founded on spatially random soil, Geotechnique, 1993, 43(4), 577-587.

[19] XIU D, Karniadakis G.E., Modeling uncertainty in steady state diffusion problems via generalized polynomial chaos, Computer Methods in Applied Mechanics and Engineering, 2003, 191 (43), 4927-4948

[20] Gray R.M., Toeplitz and Circulant Matrices: A review, Department of electrical Engineering Stanford University, 2006. 